\title{
LA FRAGMENTACIÓN DE BOGOTÁ Cómo las comunidades marginadas son cada vez más marginadas.
}

\author{
Jorge Andrés Naranjo León \\ Politécnico de Turín \\ Profesor: Arq. Giuseppe Cinà \\ j.andres.n191@gmail.com
}

\section{RESUMEN}

Este documento se basa en la hipótesis de que la ciudad posee una división estructural, esta la fragmenta en dos pedazos. La parte oriental que contiene; gracias a una tradición histórica y a factores políticos y socioeconómicos; la gran mayoría de equipamientos, las centralidades y zonas de actividad económica intensiva, los servicios y la infraestructura. La parte occidental al contrario, se convierte en una zona aislada, con una predominancia residencial con grandes carencias a nivel de infraestructura de servicios, de movilidad y equipamientos.

Se realiza un acercamiento a la problemática a través de la movilidad, y como esta esta estrechamente ligada con la desigualdad social presente en muchas ciudades latinoamericanas. Ante esto se proponen estrategias generales, que se consideran, podrían generar una revitalizar zonas de periferia.

Palabras clave: Periferia, Movilidad, Espacio público, Desigualdad social

\section{ABSTRACT}

The document is based on the hypothesis that the city is divided into two portions. An east section that due to a historical relevance and economical and political background holds most of the infrastructure, services and economically relevant zones whereas the west remains a mainly residential area with an immense need of facilities and transportation modes.

The issue is approached focusing in mobility and how it is closely linked with other issues such as social inequality; a matter highly visible in South American cities.

Then, general planning strategies are proposed from a standpoint where is believed, could help reshape peripheries into more active and dynamic areas.

Key words: Periphery, Mobility, Public space, Social inequality 
Las ciudades latinoamericanas, al estar bajo los ojos de las instituciones durante décadas se han convertido en entidades tan complejas que parece casi imposible encontrar una manera de llevarlas a un futuro menos sombrío.

El caso de los dilemas de Bogotá podría resumirse en una variedad de problemas sociales, la falta de infraestructura, la escasez de medios para responder a una crisis de vivienda permanente, la corrupción y una batalla política constante que obstaculiza una actuación efectiva del sector público en áreas donde es más necesitado. Todo lo anterior ligado entre si haciendo más difícil encontrar estrategias de desarrollo y posibles cambios.

En mi tesis de maestría; Nuevo Rincon - La expansión y diversificación de la Centralidad Suba a través de la movilidad y la renovación de la UPZ El Rincón; Se menciona uno de los aspectos clave que podría comenzar a llevar desarrollo a áreas marginadas, específicamente en la porción oeste de Bogotá; esta es la movilidad y el transporte público.

Esta es estos ya mencionados, son expuestos como detonantes del desarrollo en zonas de alta concentración de población ya que puede impulsar el cambio de modelo de ciudad de mononuclear a polinuclear.

Se sabe que es común que la actividad comercial, los equipamientos principales y las interacciones urbanas tienden a concentrarse a los bordes de los ejes principales en las diferentes escalas de una ciudad o asentamiento. (Interviniendo ciertos ejes con elementos de movilidad, transporte publico, equipamientos para el uso de la comunidad, espacio publico etc; se impulsa el crecimiento de ciertas zonas) como centralidades de segundo y tercer orden, posiblemente aumentando su relevancia a nivel zonal modificando poco a poco el modelo de ciudad. (Arismendi. 2010)

"Uno de los elementos que al introducirse generan un mayor impacto en el territorio es el desarrollo de los caminos o vías terrestres, que como puntos de comunicación e intercambio, logran establecer la dirección de crecimiento de las áreas urbanas, posteriormente será la naturaleza social la que establezca los requerimientos de las ciudades y defina su consolidación en el tiempo y el espacio a partir de su continua transformación física, social y funcional." (Villamizar-Duarte y Sánchez, 2012: 1)

(El surgimiento y potenciamiento de las centralidades de segundo y tercer orden en el occidente de la ciudad podría, no solo generar nuevos empleos, un aumento del valor del suelo, conllevando a mayor inversión a nivel público y por ende mejoramiento de la calidad urbana; sino también un crecimiento a nivel de la sostenibilidad puesto que se reduciría el numero de desplazamientos pendulares hacia otras zonas de la ciudad, disminuyendo así el trafico, y los efectos medioambientales que representa.)(GUEVARA, M.)

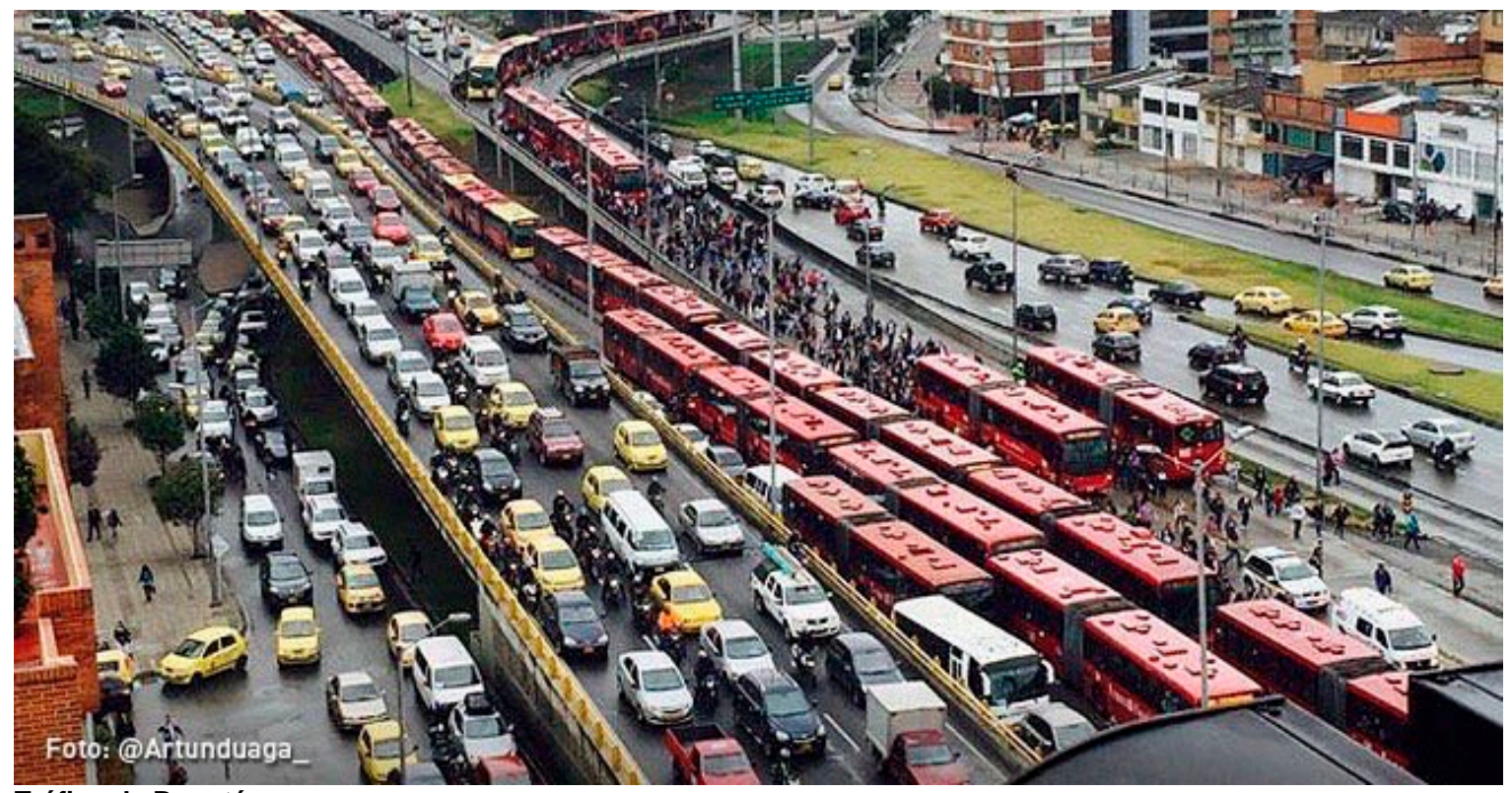

Tráfico de Bogotá

Foto tomada por @Artunduaga_18 ene. 2018 Fuente: Twitter

Se sabe que el transporte masivo es una necesidad para las ciudades, aún más ahora que los efectos del hombre en el planeta son cada vez más visibles. Sin embargo, las medidas sostenibles deben ir un paso más 
allá de la implementación de este para lograr reducir la cantidad de automóviles y sus efectos, con el objetivo de desarrollar sistema que minimice el desperdicio de energía y que use fuentes de energía sostenibles.

Este es un gran desafío para las ciudades en países en vía de desarrollo, donde existe un alto porcentaje de asentamientos informales en las zonas de periferia. Zonas que a pesar de la reglamentación por parte de los gobiernos locales continúan expandiéndose.

Esto hace más evidente la falta de la inversión pública y obstaculiza la implementación de tales estrategias de movilidad.

La implementación de infraestructura de transporte masivo requiere en estos casos una inmensa cantidad de recursos puesto que deben enfrentar aspectos técnicos, sociales y económicos que chocan con este asunto.

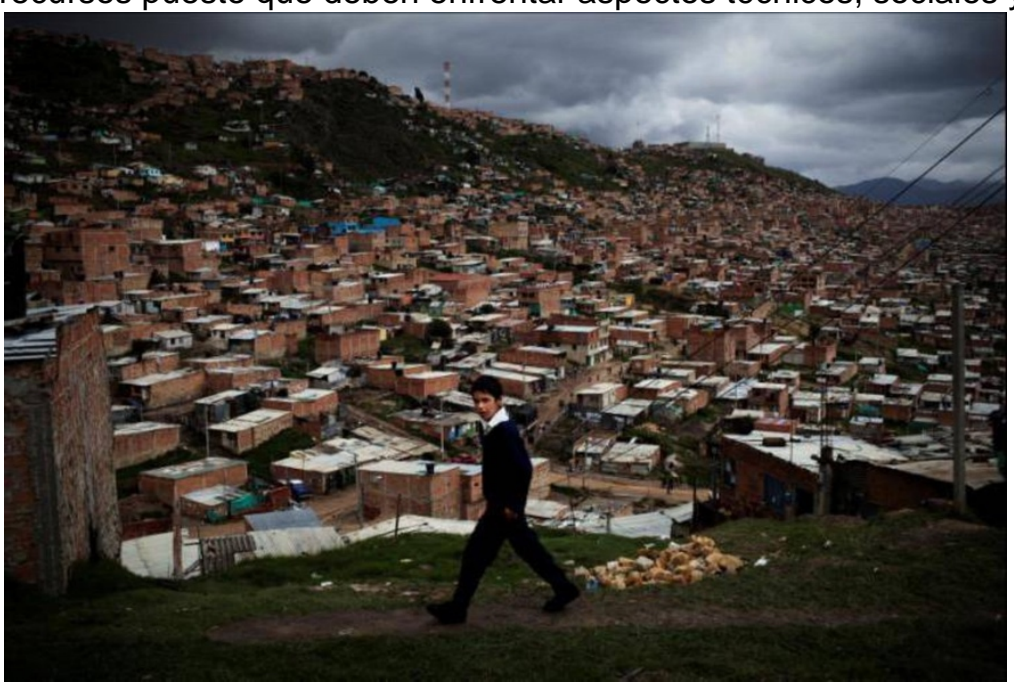

Es en este último argumento en el que se podría afirmar que la movilidad en algunos casos es un reflejo de las problemáticas sociales en las ciudades. Un ejemplo de esto son los barrios marginados donde las carreteras no están pavimentadas o donde el transporte público no llega. Este segundo elemento es válido también para comunidades extremadamente ricas, aisladas del tejido urbano de las ciudades donde el automóvil privado es un requisito para el acceso, excluyendo así a las personas con un estatus socioeconómico limitado. Esto convierte a la movilidad hasta cierto punto en un Ciudad Bolívar y la vivienda informal Tomada el 19 ene. 2017 de:

http://www.acnur.org/fileadmin/_processed_/csm_09_03_c822df9df3.jpg elemento político, determinando el acceso de la población a ciertas áreas de la ciudad.

Esto sin contar problemáticas antropológicas arraigadas en los países relacionadas a la movilidad. Inconscientemente, las personas en países con gran desigualdad social perciben el transporte público como un tipo de transporte de clase media I baja, factor que crea un estigma en el resto de las personas haciéndolo menos atractivo para el público en general.

Esto ultimo, se evidencia en el modo como la publicidad automotriz llega a los consumidores, y como la población percibe tener un vehículo privado, de uso familiar o personal, convirtiéndose en símbolo de status y crecimiento económico.

Esto significa que los gobiernos necesitan desarrollar incentivos hacia todo tipo de clase social, no solo mediante la creación de políticas agresivas contra el uso de vehículos particulares; o de combustibles contaminantes aumentando impuestos, reduciendo cantidad de estacionamientos, etc; sino creando sistemas más atractivos que generen el interés de los ciudadanos para su uso.

A nivel urbano, el rol de la movilidad es determinante en el desenvolvimiento de las dinámicas de la ciudad mediante un modelo especifico, sea centralizado, lineal, poli-nuclear, etc.

La movilidad está determinada por una infraestructura vial, un conjunto de modos de transporte públicos y privados, conexión con el espacio público, aceras, plazas, parques, etc., nodos que, articulados con las actividades llevadas a cabo, determinan los flujos.

(Gracias a este último es posible ver el surgimiento de áreas comerciales predominantes, que luego forman sectores y crean subflujos y formas de transitar y vivir la ciudad.

Dependiendo de la movilidad, los ciudadanos comienzan a ver y vivir la ciudad a su manera. Se podría inferir que la movilidad tiene el poder de modificar cómo la gente ve la ciudad, cómo la vive, cómo se mueve dentro de ella.) (JOHNSON, 2003)

Sin embargo, la movilidad no puede ser el foco principal en una ciudad, ya que puede volverse invasiva, abusando de sus usuarios, los ciudadanos. Es cierto que una ciudad como Bogotá no puede enfocarse solo en la movilidad ya que ésta no es un asentamiento de paso sino que es dentro de ella que se desenvuelven todo tipo de actividades. Sin embargo siendo ésta una posible barrera para el crecimiento, y para la resolución de otras problemáticas, es necesario crear estrategias para impulsar si mejoramiento. 
La situación actual muestra a Bogotá con un sistema que no responde adecuadamente a la dinámica de la ciudad; permitiendo el acceso a las periferias, a las áreas de servicios, a las residencias; generando otros problemas, deterioro, subutilización de zonas de alto potencial y la disminución de la calidad de vida, entre otros.

La movilidad en una ciudad como Bogotá ya no puede determinarse estrictamente por el espacio para el uso de vehículos privados. Las consecuencias de su uso, tanto desde el punto de vista medioambiental así como en cuestiones de desperdicio energético, de tiempo y de productividad, son muy notables. Las medidas constantes para reducir sus efectos continúan ejecutándose en un constante proceso de prueba y error.

Por otro lado, es obvia la necesidad de implementar sistemas de transporte masivo para optimizar el uso de las vías principales, conectando las zonas de servicios y las zonas económicamente intensivas con las áreas residenciales; sin embargo, Bogotá continúa luchando para ejecutar tales sistemas no solo por razones de financiamiento sino por el choque de intereses privados, corrupción y una visión prospectiva sesgada que no ha llevado a nada más que a la obsolescencia de intervenciones previas y del sistema existente mientras la población sigue aumentando.

En Bogotá, Transmilenio evidentemente, se ha vuelto obsoleto en relación con las demandas de una ciudad con aproximadamente 10 millones de personas, residentes y viajeros, que necesitan movilizarse a diario; y que están obligados a hacerlo utilizando solo un sistema de autobús articulado de 120 personas que no solo no cubre la totalidad de la ciudad, sino que no tiene la capacidad en sí misma para hacerlo.

Una ciudad capital como Bogotá no solo debe haber implementado modos de transporte de alta capacidad como el metro, sino que también haber consolidado un sistema completo que incluye modos más sostenibles como tranvías, trenes suburbanos, cables aéreos, autobuses eléctricos además de Transmilenio y su flota alimentadora.

Sin un sistema más accesible para todas las áreas de la ciudad, la ciudad seguirá decayendo, produciendo más y más problemas sociales.

La ciudad a lo largo de su historia le ha huido a la posibilidad de implementar un sistema intermodal, monopolizando el sistema basados en intereses particulares y en políticas corruptas. Desde la desaparición del tranvía en los años 50, posteriormente del trolebús, y por último de los operadores de buses no articulados.

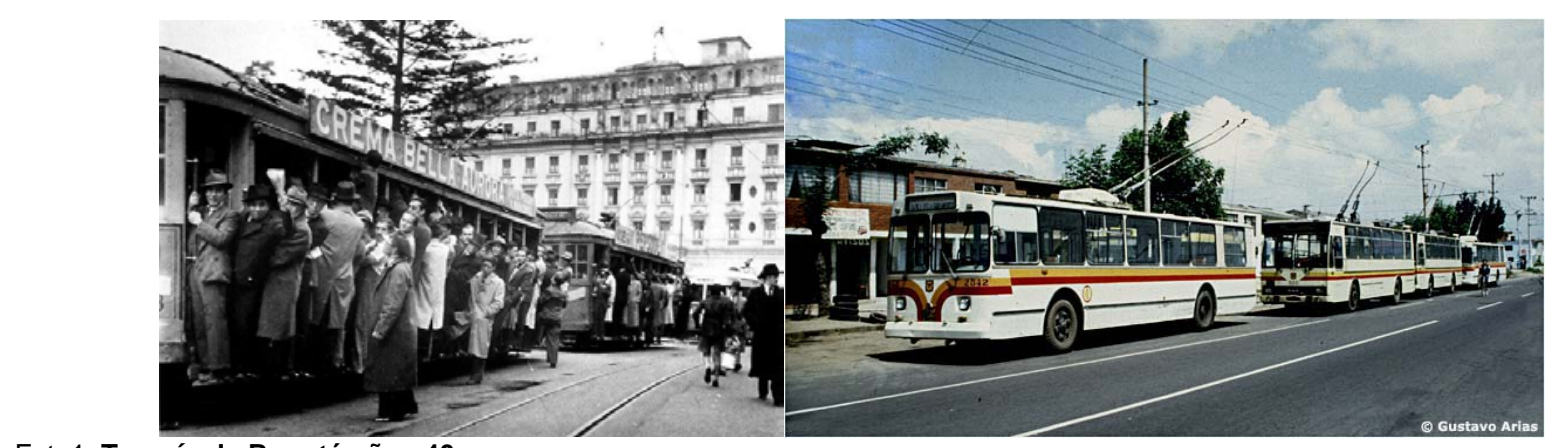

Foto1: Tranvía de Bogotá años 40

Tomada de:

http://www.rcnradio.com/audios/que-sucedia-en-colombia-durante-la-segunda-guerra-mundial-un-investigador-le-cuenta/ Foto 2: Trolebus 1985

tomada de: http://static.panoramio.com/photos/large/49695252.jpg

Hoy en día, ante el sistema fallido de buses (SITP y Transmilenio) la ciudad clama la construcción de un metro, sin embargo, aún existen dudas por parte del gobierno sobre la eficiencia y el modo de implementación del tren subterráneo, o en el caso de Bogotá, elevado. Esto, en relación con su sobresaturación como ocurre ahora con el Transmilenio; y si podría realmente ayudar a disminuir la congestión en ciertos ejes.

Al mismo tiempo, dichos sistemas deberían ubicarse en ejes de alto tránsito, donde en muchos casos se encuentra actualmente Transmilenio. Esto parece crear un conflicto ya que podría ocurrir una posible subutilización de uno u otro, por lo que sería inviable desde un punto de vista económico y de gestión.

Sin en ciudades del mundo con altos niveles de población y una gran demanda, como Nueva York, París, Berlín, San Petersburgo, como muchas otras, se usan múltiples modos de transporte en los mismos corredores principales. Esto permite, en ciertos casos, no estar constantemente sobresaturados. También crea una especie de comodidad en el sistema para hacerlo atractivo y funciona como una medida para mitigar el uso de automóviles particulares en ciertas partes de la ciudad.

Con relación a la intervención del metro en Bogotá se considera que es necesario evaluar las afectaciones a nivel urbano, y como en lugar de tejer ciudad, podría crear rupturas o convertirse en ejes de deterioro. El 
diseño y la intervención directa en el sitio deben articular el contexto urbano entre si; los núcleos funcionales, el espacio público; y sobre todo el usuario y la forma en que entrará a hacer uso de esta.

Los sistemas de trenes metropolitanos subterráneos no crean brechas que puedan fragmentar la ciudad, sino que cumplirá su función de conector, permitiendo además un flujo continuo y fácil para el desarrollo de otras actividades en la superficie.

Sin embargo recientemente, el gobierno local obstinadamente y con excusas de ahorro en costos de obra y de financiación esta llevando a cabo el proceso para la construcción de un metro elevado. Proceso que ha sido criticado por académicos e incluso anteriormente por el que es hoy cabeza del gobierno, el alcalde Enrique Peñalosa.

Las criticas sobre los efectos colaterales de este tipo de implementación de un sistema que debería ayudar a solucionar problemáticas de movilidad argumentan que ahondarían en otro tipo de afectaciones a nivel urbano, social y económico.

Así mismo dentro de los posibles sistemas a implementar en la ciudad, existe el tranvía, modo que por diferencias de ideologías políticas se ha propuesto y rechazado en múltiples ocasiones pero que ayudaría a suplir la demanda, son sostenibles y no producen una brecha urbana tan drástica como lo hacen otros modos.

La ruptura urbana comienza cuando una intervención crea vacíos urbanos o espacios intersticiales (terrenos vagos) sin uso, función o atractivo y donde los peatones no tienen acceso. Un ejemplo de eso son las grandes brechas junto a la Estación Escuela Militar sobre la Calle 80 y la Carrera 30 o la misma al lado de la estación Calle 26 sobre la Avenida Caracas y la intersección y los túneles de la Avenida 26. Y lo mismo en los túneles de la avenida Carrera 30 y sus múltiples túneles y puentes. Todas estas muestran formas de resolver ciertos problemas de movilidad, creando soluciones fáciles pero que afectan otros aspectos a nivel urbano y social.



Deprimido de la Calle 94 Calle 26 Vacío urbano de la inexistente Estación Central Fotografías tomadas de Google Street view el 15 de Febrero de 2018

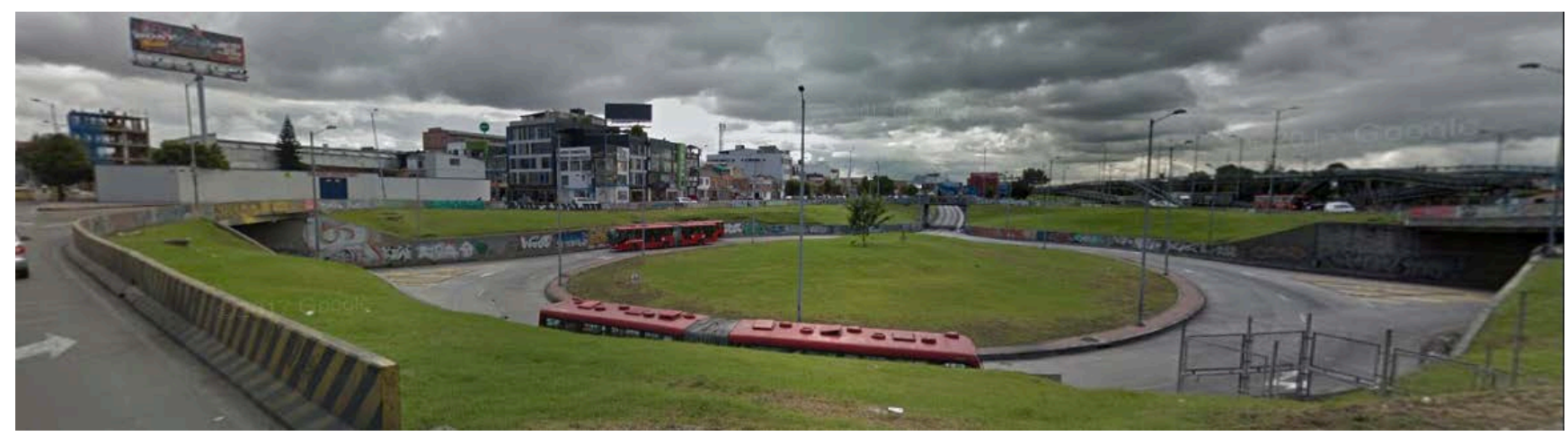

Vacío urbano Escuela Militar

Fotografías tomadas de Google Street view el 15 de Febrero de 2018

Estos escenarios muestran cuán importante es articular tales intervenciones con el espacio público y otras funciones para enmendar las brechas, como el parque Bicentenario en Bogotá o la estación intermodal, ambas en zonas de deprimidos en la calle 26 Avenida. 


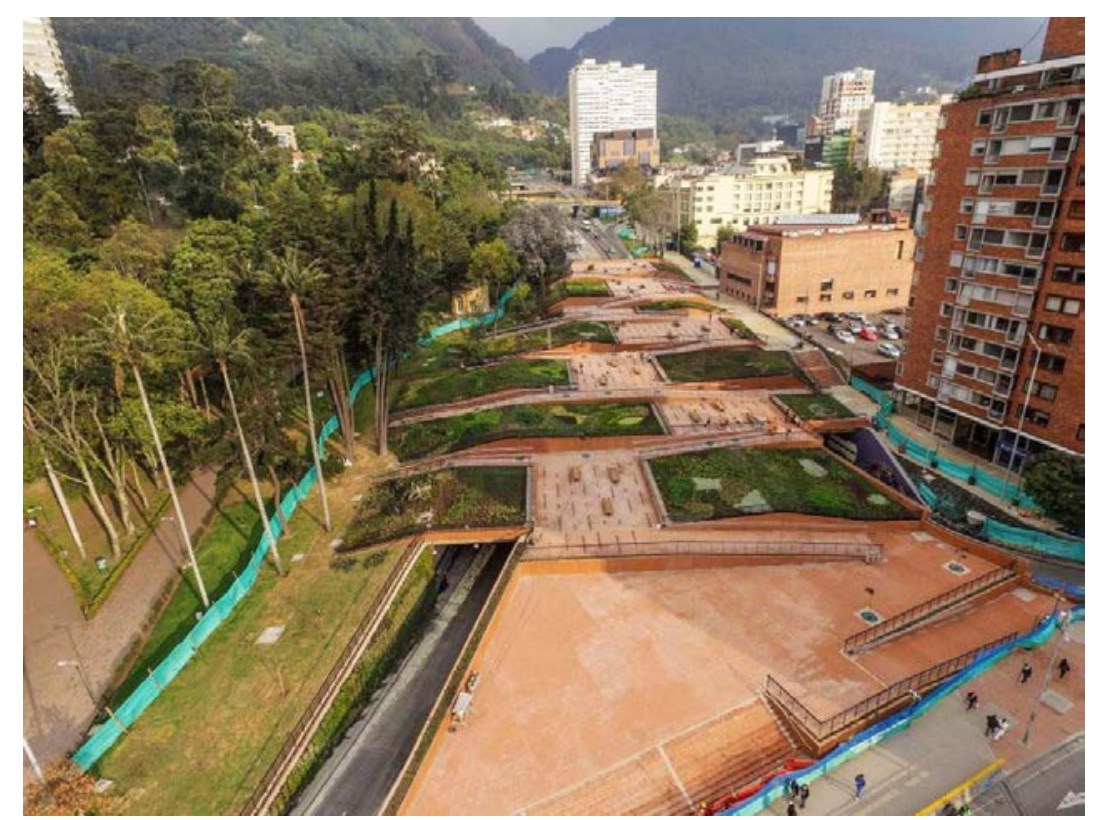

Parque Bicentenario. Tomada de:

http://www.greenroofs.com/projects/parque_bicentenario/parque_bicentenario9.jpg

Como un factor adicional, Bogotá tiene un aumento en los modos de transporte informal, que quiera o no el gobierno, ayudan a resolver problemas de movilidad que parecían incapaces de solucionar. Microbuses, taxis compartidos y bicitaxis que hacen circuitos por los vecindarios donde el transporte "formal" no va. Sin embargo, continúan siendo ilegales.

Es posible que estos modos no puedan garantizar los mismos niveles de seguridad que los demás, sin embargo funcionan a un precio justo o más bajo que los convencionales, manteniendo un servicio de buena calidad que crea subsistemas sostenibles y eficientes además de crear empleos.

El surgimiento de este tipo de sistemas informales continuará siendo una parte de la ciudad hasta que la ciudad garantice el acceso a cada área de la ciudad.

Y aún así, cuando se implemente, el cambio constante y la expansión de la ciudad crearán nuevos modos, nuevos sistemas, y continuarán respondiendo a las nuevas necesidades. Es lo que mantiene viva a la ciudad. 


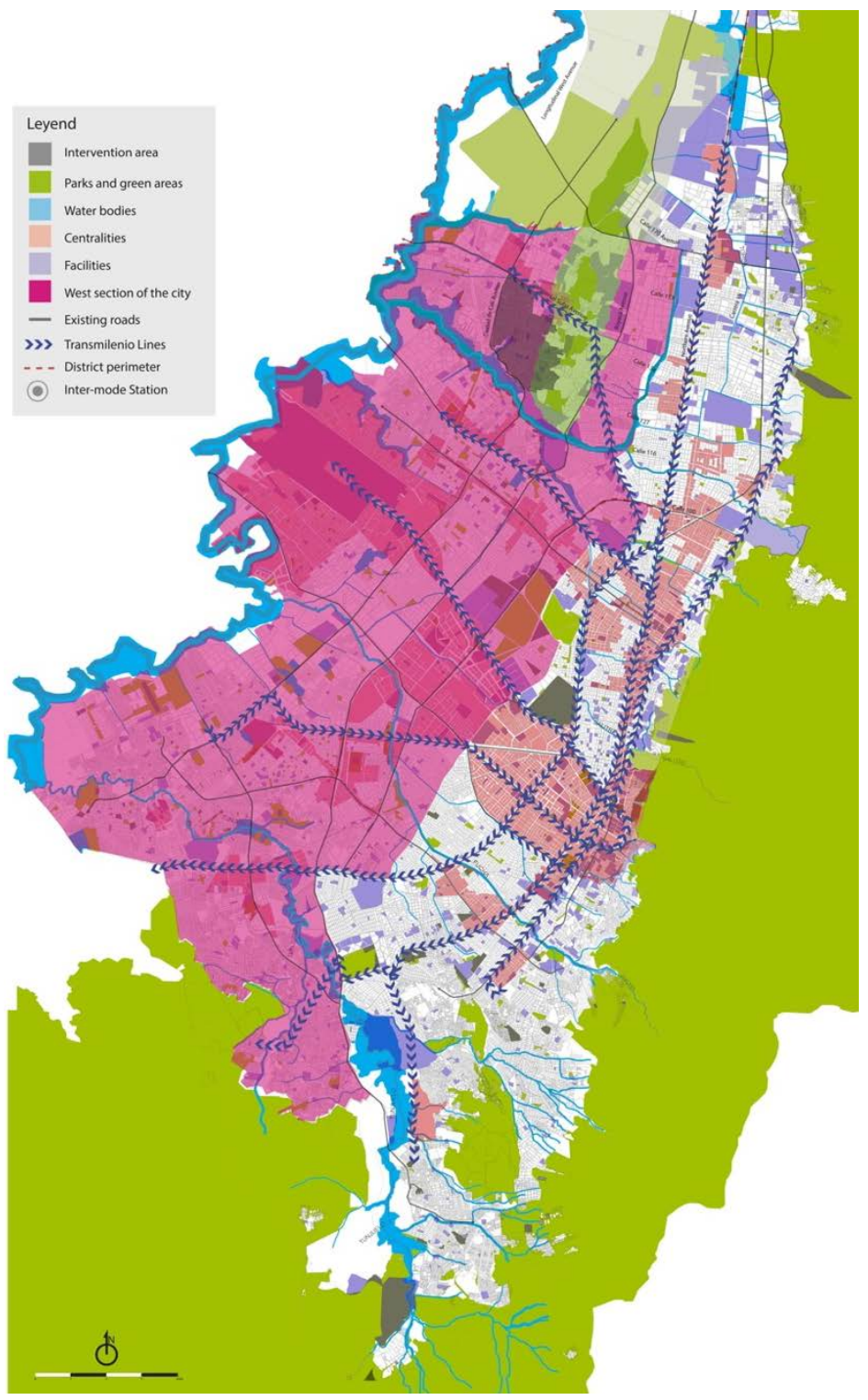

La fragmentación de Bogotá:

Elaboración propia. 2017

y sobre todo ciertas centralidades. Esto es lo que sucede con las centralidades en el oriente.

Como estas últimas tienen los medios para responder a la mayoría de las necesidades de la población, dichas áreas siguen recibiendo inversiones públicas que acentúan el deterioro de otras centralidades en el occidente que en deberían aliviar la demanda de las centralidades principales.

El hecho de que las personas necesiten desplazarse desde el occidente hacia el oriente no solo crea un colapso en el sistema de movilidad sino también en otros sistemas. Es entonces donde se evidencia una sobre-saturación en los equipamientos de salud y educación, puesto que los que se encuentran al occidente no tienen la capacidad de responder a la demanda poblacional, ni la posibilidad de prestar servicios específicos.
En el momento en que se implementen nuevos sistemas formales, sería apropiado formalizar tales modos "ilegales", en un sistema más organizado, integrado a los demás, con garantías de seguridad y vehículos mejor fabricados con la misma identidad sostenible.

Sin embargo, Bogotá se convierte en una ciudad bastante particular cuando se observa que los subsistemas que surgen no solo son visibles en las áreas periféricas de la ciudad, sino que se pueden encontrar en una gran parte de la ciudad, el oeste.

En mi tesis se defiende la hipótesis de que la ciudad está dividida en dos partes principales, el este y el oeste, donde el este posee la mayor parte de la infraestructura y los servicios, y el oeste es una zona residencial relegada.

La estructura principal de la ciudad está diseñada para tener múltiples centralidades con servicios para responder a los habitantes que los rodean, sin embargo, no cumplen con las necesidades actuales de la población, lo que les obliga a viajar, saturar otros sistemas

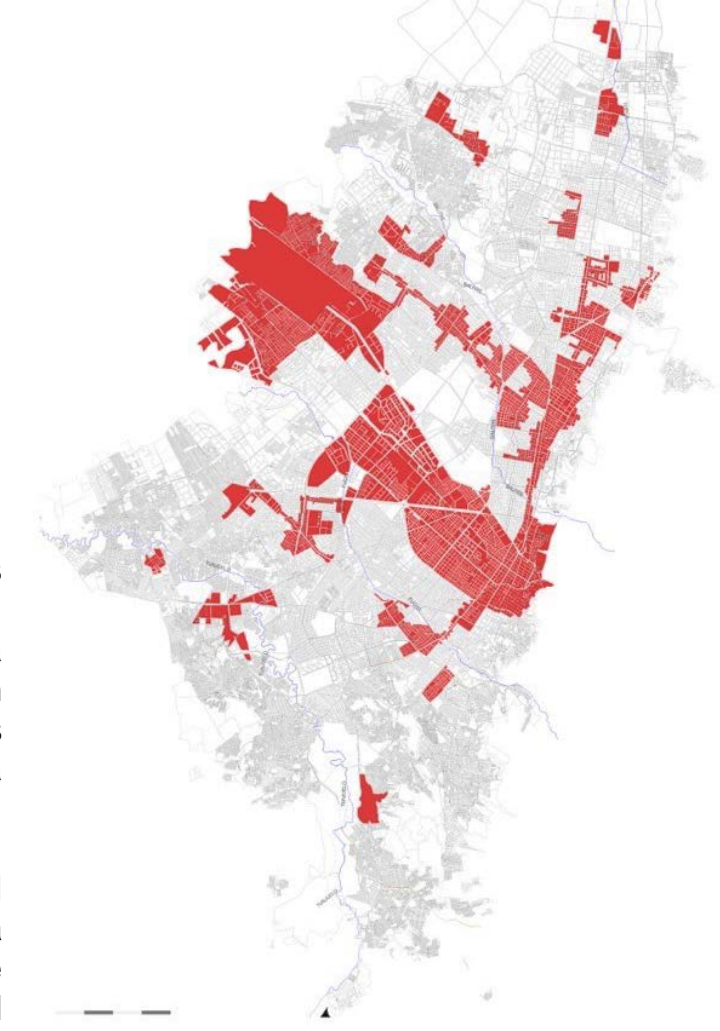

Centralidades de Bogotá: Elaboración propia. 2013 Fuente: SDP

En otras palabras, Bogotá funciona como un modelo mono-nuclear con múltiples falsos núcleos internos. Está claro que muchos de los barrios de las localidades del occidente tienen un origen informal, por lo tanto, un contexto no planificado. 
La razón de tales altas tasas de informalidad está relacionada con el elevado número de inmigrantes que huyeron de la guerra en el campo buscando seguridad y nuevas oportunidades en la ciudad, esto se refleja en el crecimiento exponencial de la población de la ciudad en la segunda mitad del siglo XX. Pasando de 330,000 en 1938 a 3'992.200 en 1992 y casi 10 millones en 2017.

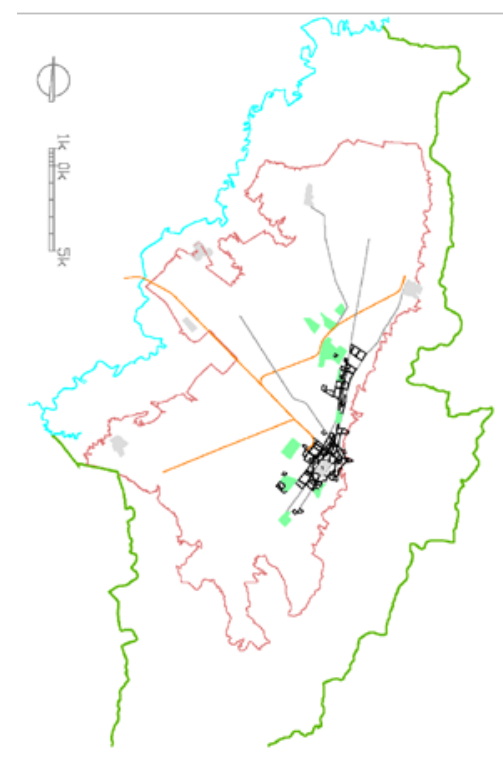

1930

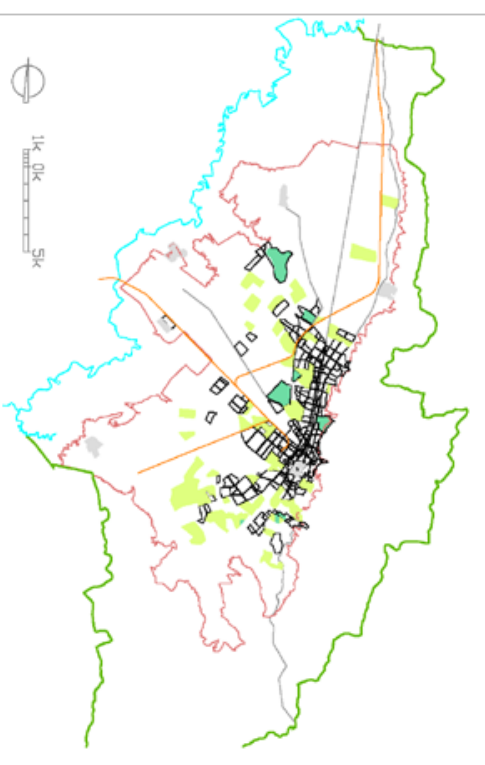

1950

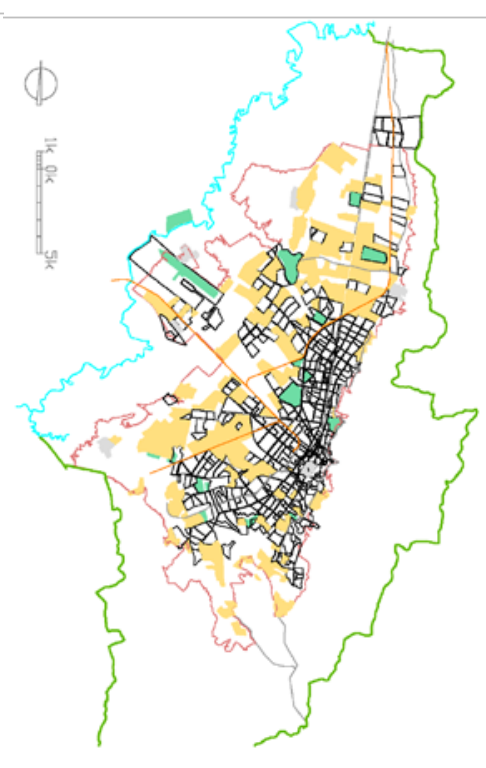

1970

Expansión urbana Bogotá 1930 - 1970.

Fuente: Arq. Ernesto Moure - Pontificia Universidad Javeriana. 2014

Este déficit de vivienda, aún latente, sumado a la pobreza extrema provocada por la guerra produjo el surgimiento de viviendas de autoconstrucción que en muchos casos estaban ubicadas en zonas de riesgo o de protección ambiental, mal construidas, sin tomar en cuenta factores como elementos sismorresistentes; utilizando materiales de baja calidad y estableciendo parcelas pequeñas con espacios inhumanos.

La expansión continua de una periferia no planificada, la aparición de asentamientos ilegales se convierte entonces en un problema más complejo para los planificadores urbanos y para el gobierno que necesita encontrar nuevas formas de proporcionarles transporte y servicios.

El aumento de los asentamientos en zonas de riesgo ambiental en la periferia hace que sea aún más difícil implementar nuevos medios de movilidad. Tales desafíos se convierten en una cuestión técnica, económica y social.

Cuando se habla del occidente de la ciudad, se debe entender que el ésta se convirtió en parte de Bogotá a través de un proceso de conurbación consecuencia de una expansión similar a una mancha e aceite.

Este proceso se aceleró de manera exponencial puesto que por razones socio-políticas; mas explícitamente por el surgimiento de la violencia y el conflicto armado; familias enteras se vieron obligadas a dejar el campo para ir a las ciudades donde estarían a salvo.

La expansión no planificada de la periferia reflejaría la carencia de políticas de emergencia, carencia de vivienda y la negligencia por parte del gobierno para atacar la raíz del problema.

Las personas que llegaron se asentarían ilegalmente en las afueras de la ciudad, apropiando el territorio y en muchos casos invadiendo reservas naturales, en la mayoría de los casos en lugares de riesgo ambiental.

Estas zonas periféricas se mantuvieron en la ilegalidad por décadas, careciendo en muchas ocasiones de servicios públicos básicos, en vías pavimentadas y en equipamientos y espacio público.

(La carencia de este tipo de infraestructura de servicios, sumado a el contexto de violencia y falta de presencia del estado propició el surgimiento de problemáticas sociales, y el rechazo del resto de la población que tuvo los medios para establecerse en el territorio en condiciones adecuadas y en zonas aprobadas por el estado. Hasta el día de hoy en las zonas de periferia se ha mantenido un estigma perpetuando los problemas sociales como la violencia, la inseguridad, la ilegalidad, entre otros.) (MCKEE, 2013)

Este es también el caso no solo de muchos países en desarrollo en América Latina, África y el Pacífico Sur, sino también en países europeos donde las periferias de las grandes ciudades se convierten en guetos donde 
los ciudadanos de bajos recursos, los inmigrantes y las minorías son marginadas, no solo en aspecto político pero en un aspecto urbano y arquitectónico.

Las periferias, en un contexto socioeconómico, se clasifican más allá de las características geográficas y la ubicación que en realidad juegan un papel importante al momento de entender la división social actual.

Podría decirse que las áreas en parcelas con altos niveles freáticos, cerca de las zonas de peligro natural; áreas propensas a inundaciones, deslizamientos de tierra, etc .; están inclinadas a ser utilizadas por las clases más bajas, mientras que las áreas rodeadas por un contexto natural agradable, bosques o colinas con terrenos con una gran capacidad de carga son más utilizados por los grupos ricos para vivir.

Ambos terrenos ubicados en una periferia; en algunos casos incluso, presentando similitudes micro zonales a nivel urbano como el "conjunto cerrado".

El factor clave que los diferencia es la cantidad de metros cuadrados por habitante, los materiales y acabados usados.

(Curiosamente, ambos se encuentran comúnmente en periferias privadas de transporte, instalaciones públicas e incluso de comercio de suministros básicos.

Para ambos casos construir en este tipo de terrenos significa un precio más bajo del suelo y parcelas listas para ser compradas e intervenidas; que no plantean ninguna dificultad en cuanto a procesos burocráticos como el cambio de uso y la adquisición de licencias; problemas que se encuentran comúnmente en el interior de la ciudad.) (JOHNSON. 1974)

"Contradictoriamente, mientras los grupos de habitantes de menores ingresos deben ocupar la periferia alejada del centro urbano (según la tendencia histórica), a su vez los habitantes de mayores ingresos dentro de un proceso de -huida- de la Metrópoli pretenden localizarse al exterior de la ciudad en busca de mejores condiciones de vida y asistidos por la tecnología. Ambas formas de ocupación opuestas en sus características físicas y sociales tienden a conquistar el territorio suburbano. En ambos casos existe dependencia del uso de medios de transporte motorizados para acceder a las centralidades urbanas, ya sea en modos masivos o individuales." (VILLALOBOS, 2012: 13)

Si el modelo de la ciudad se mantiene tal cual, la medida inmediata de mayor urgencia sería proporcionar el transporte adecuado a la mano trabajadora que vive en el oeste. Lo cual no está sucediendo

El oeste de la ciudad está conectado por solo 4 líneas de Transmilenio, apenas respondiendo a la demanda.

Esa situación revela, entonces, la necesidad de establecer un modelo de ciudad diferente que podría aliviar no solo los problemas de movilidad, sino a mayor escala, una mejora general de la calidad de vida.

Por lo tanto, se propone un modelo poli-nuclear, donde la mayoría de los servicios se puedan encontrar en toda la ciudad.

"En el actual contexto social y económico, el estudio sobre la del borde como espacio físico identificable en los territorios urbanos, conlleva a la observación de procesos urbanos de diversa naturaleza como la expansión, la densificación de áreas centrales, la consolidación de nuevas centralidades, la incorporación de áreas informales, la articulación funcional y física con áreas rurales al interior de las ciudades, las relación funcional y física con otros territorios regionales, etc." (Villamizar-Duarte, Luna Sánchez. 2012: 44) 


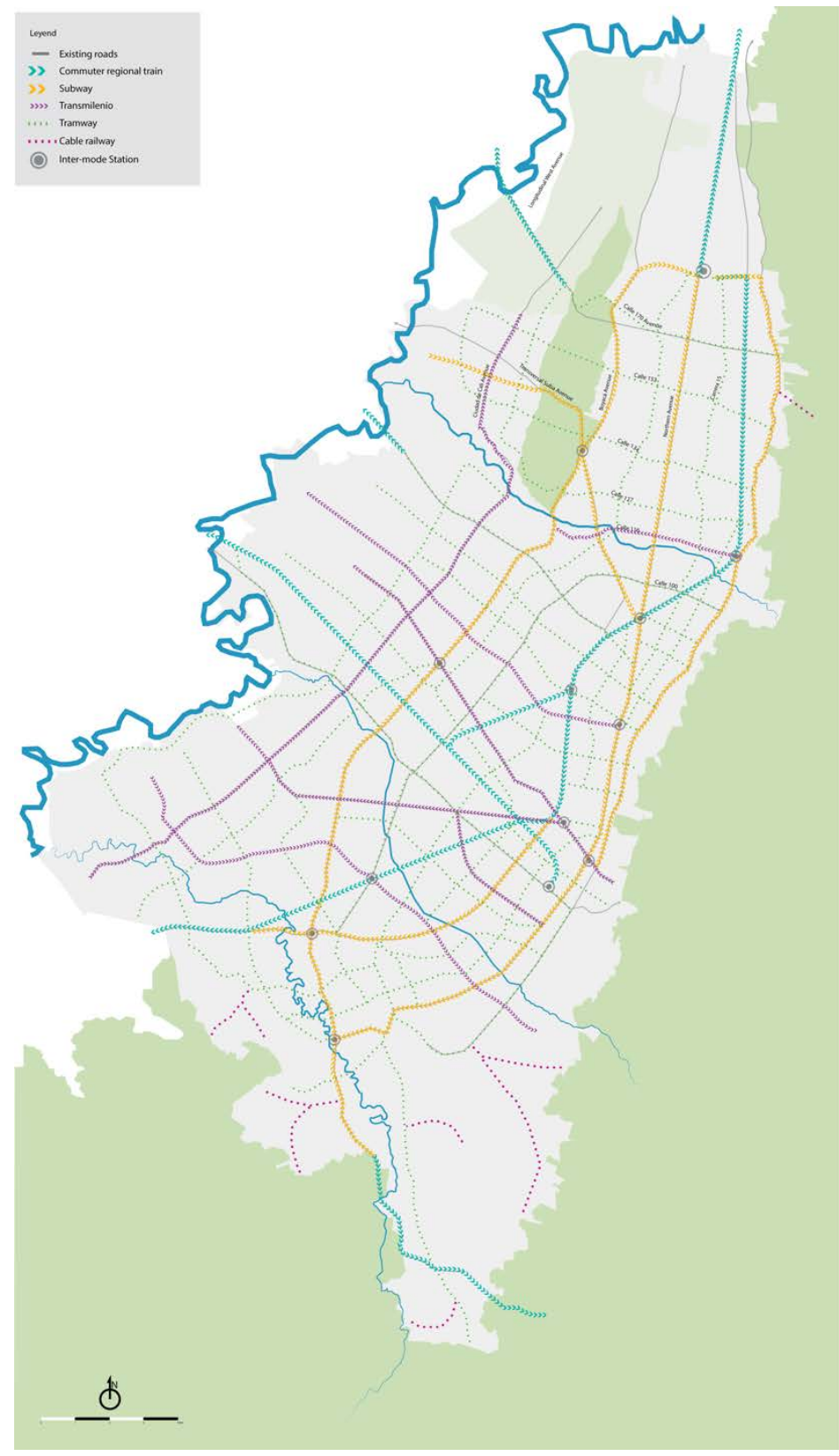

Propuesta metropolitana de movilidad para Bogotá.

Elaboración propia.

Tesis Magistral: Nuevo Rincón - La expansión y diversificación de la Centralidad Suba a través de la movilidad y la renovación de la UPZ EI Rincón

eventos públicos culturales y sociales.

Un espacio público sigue siendo un elemento relevante en las ciudades, por lo que su implementación en áreas que carecen de él es realmente importante.

Dichos espacios permitirán el surgimiento de nuevos lugares de encuentro para la interacción humana, el ocio y los nuevos mercados, sin dejar de lado el hecho de que socialmente impulsarían el arraigo de las personas al lugar y crearían un sentido de comunidad.

Una estrategia de este tipo comenzaría a transformar la ciudad desde la escala local, sin enfocarse en el planeamiento urbano desde las instituciones sino desde las comunidades.

Este urbanismo bottom-up tendría efectos significativos puesto que generaría un mayor atractivo en puntos estratégicos, que unidos con estrategias de movilidad, de infraestructura de servicios y de vivienda podría impulsar el crecimiento de las centralidades de segundo orden, generando mayor inversión pública y privada y un mejoramiento integral del occidente de la ciudad y de la ciudad completa.)(QUINTERO. 2009) 


\section{Bibliografía:}

GUEVARA TRIANA, M. (2011). Aplicación de los planes parciales como concreción del modelo de ordenamiento territorial en Bogotá: Hacia una propuesta metodológica. Maestría de Planeación urbana y regional. Bogotá: Pontificia Universidad Javeriana.

VILLALOBOS CAMARGo, C. (2012). Aporte al concepto teórico del suburbano según la dinámica de producción urbana en el proceso de crecimiento de Bogotá hacia el primer anillo de periferia. Maestría de Planeación urbana y regional. Bogotá: Pontificia Universidad Javeriana

ARISMENDI ABRIL, S. (2010). Tesis de grado. Centro de Negocios y servicios como apoyo a la reactivación del centro de Bogotá. Bogotá: Pontificia Universidad Javeriana.

QUINTERO, O. (2009). Ciudad - Región y el proyecto regional, una mirada sobre la reconfiguración territorial en Colombia. Maestría de Planeación urbana y regional. Bogotá: Pontificia Universidad Javeriana.

ISAZA GUERRERO, J. (2008). Conurbación y desarrollo sustentable: una estrategia de intervención para la integración regional. Caso primer anillo metropolitano Bogotá - Sabana de Occidente. Maestría de Planeación urbana y regional. Bogotá: Pontificia Universidad Javeriana.

VILLAMIZAR- DUARTE, N., LUNA SÁNCHEZ, J. (2012) Bordes urbanos: una pregunta desde el crecimiento de las ciudades hacia la concepción de una categoría para el análisis y la proyección de territorios urbanos. Bogotá: Universidad Nacional de Colombia.

JOHNSON, J.H. (1974). Suburban growth: Geographical processes at the edge of the western city. Aberdeen: Aberdeen University Press.

BAZANT S, J. (2001). Periferias Urbanas. Expansión urbana descontrolada de bajos ingresos y su impacto en el medio ambiente. México. Ed. Trillas.

DEL CASTILLO, J. (2001) La región polinuclear un futuro posible para Bogotá y la Sabana. Revista Bitácora urbano territorial Universidad Nacional de Colombia (Bogotá). 005, 9-15.

- Cartografía de Bogotá y su estructura territorial. http://webidu.idu.gov.co:9090/pmb/opac_css/index.php?lvl=categ_see\&id=3639

CLICHEVSKY, N. (2003) Pobreza y acceso al suelo urbano. Algunas interrogantes sobre las politicas de regularizacion en America Latina. Santiago de Chile: CEPAL.

JOHNSON, S. (2003) Emergence: The Connected Lives of Ants, Brains, Cities, and Software. New York: Simon and Schuster Editorial.

\section{Fuentes Electrónicas:}

MCKEE, A.J. (2013) Broken windows theory: academic theory -dam J. - Encyclopedia Britannica https://www.britannica.com/topic/broken-windows-theory (Consulta: 13/06/2017) 